\title{
Convexification Technique and Portfolio Optimization
}

\author{
Cristinca FULGA ${ }^{1,2}$ \\ ${ }^{1}$ The Bucharest University of Economic Studies, \\ 6, Piata Romana, 010374 Bucharest 1, Romania, \\ fulga@csie.ase.ro \\ 2 "Gheorghe Mihoc-Caius Iacob" \\ Institute of Mathematical Statistics and Applied Mathematics of Romanian Academy, \\ 13, Calea 13 Septembrie, 050711 Bucharest 5, Romania
}

\begin{abstract}
In this paper, a general transformation method which converts a nonconvex optimization problem to an equivalent problem with better properties is proposed. Under certain assumptions, the local convexity of the Lagrangian function of the equivalent problem is guaranteed and thus the class of optimization models to which dual methods can be applied is extended. Practical classes of problems where the proposed method can be applied are given. They include the class of portfolio selection models. Numerical examples illustrate the main results.
\end{abstract}

Keywords: Nonconvex optimization; local convexification; Lagrangian function, portfolio optimization, efficient frontier.

\section{Introduction}

Convexity is one of the most frequently used hypotheses in optimization theory and certainly the most beneficial property in the practice of optimization because the convexity of an optimization model ensures global validity to properties otherwise only locally true. But in real life problems, the hypothesis of convexity is not very often fulfilled. The primal-dual method has been one of the most efficient solution algorithms in solving constrained optimization problems, see for example Lasdon [8], Luenberger [13]. The success of the primal-dual method depends on the local convexity of the Lagrangian function at the optimal solution of the problem. Several convexification schemes have been proposed in the literature to extend the primal- dual method for certain nonconvex problems, see for example Bertsekas [1], Li [10] and [11], $\mathrm{Xu}$ [21].

In this paper we propose a convexification technique for a general differentiable nonlinear programming problem with inequality constraints. We begin by giving some examples which illustrate the usefulness of the convexification techniques in practical situations of portfolio optimization.

\section{Examples of nonconvex models for portfolio optimization.}

The modern portfolio theory trades off the risk and expected return of a portfolio, Markowitz [15]. The classical Mean-Variance (MV) portfolio model is $\min _{x \in X}\left(-E(R), \sigma^{2}(R)\right)$, where the notations used are: $n$ the number of securities available, $r: \Omega \rightarrow \mathbb{R}$ the random vector of the asset returns $r(\omega)=\left(r_{1}(\omega), \ldots, r_{n}(\omega)\right)^{T}, \omega \in \Omega$ or simply $\mathbf{r}$ (we use bold symbols for vectors), $R=R(\boldsymbol{x}, \boldsymbol{r})=\boldsymbol{x}^{T} \boldsymbol{r}$ the return of the portfolio $\boldsymbol{x} \in X, X=\left\{\boldsymbol{x} \in R^{n} \mid \boldsymbol{x}^{T} \boldsymbol{1}=1, \boldsymbol{x} \geq \mathbf{0}\right\}$ the feasible set, $\mu_{i}=E\left(r_{i}\right)$ estimates of each asset expected return and the variance $\sigma_{i}^{2}=\sigma^{2}\left(r_{i}\right)$, correlation coefficient of any two assets $\rho_{i j}=\frac{\operatorname{cov}\left(r_{i}, r_{j}\right)}{\sigma_{i} \sigma_{j}}$, $i, j=\overline{1, n}$, the expected return and the variance of the portfolio $E(R)=\boldsymbol{\mu}^{T} \boldsymbol{x}$ and $\sigma^{2}(R)=\boldsymbol{x}^{T} \boldsymbol{\Sigma} \boldsymbol{x}$ where $\quad \boldsymbol{\mu}=\left(\mu_{1}, \ldots, \mu_{n}\right)^{T}, \quad \Sigma=\left(\Sigma_{i j}\right)_{1 \leq i, j \leq n}$, $\Sigma_{i j}=\operatorname{cov}\left(r_{i}, r_{j}\right), \rho_{i i}=1, i, j=\overline{1, n}$. The efficient frontier set is defined as the set of efficient portfolios. An efficient portfolio is a portfolio whose expected return cannot increase unless its risk represented here by its variance increases as well. But determining the set of efficient portfolios is just the first step of the portfolio selection procedure. The second step is the actual selection of one optimal portfolio out of the entire efficient frontier corresponding to the specific investor's preference structure. In order to determine an optimal portfolio corresponding to a given degree of risk aversion within the $M V$ approach, we consider the $M V$ utility function $(a>0$ and $b \geq 0)$ : $U_{a, b}(\boldsymbol{x})=a E(R)-b \sigma^{2}(R)$. The optimal portfolio corresponding to the investor's degree of risk aversion $\beta=\frac{b}{a} \geq 0$ is the solution of the problem, see Markowitz [16]: 


$$
\max _{\boldsymbol{x} \in X} U^{M V}(\boldsymbol{x})=E(R)-\beta \sigma^{2}(R) .
$$

The covariance matrix $\boldsymbol{\Sigma}$ is positive semi-definite, $x^{T} \boldsymbol{\Sigma} x$ represents the variance of the portfolio $\boldsymbol{x}$. But in practice we work with real data and it is not rare the case when the real data have missing values. In this case, one can estimate a covariance matrix by considering the matrix that will be called NAN - covariance matrix and denoted here by $\tilde{\Sigma}$ which is constructed in a simple and natural way starting from the rate of return matrix by ignoring the terms containing unknown return values (NAN is the Matlab convention for Not a Number). Unfortunately, the positive semi-definiteness of the resulting matrix $\tilde{\Sigma}$ may not be assured. The immediate consequence is the lack of convexity of the objective function of the optimization problem even in this case when the optimal decision is determined based on solely two numbers: the mean and the variance of the portfolio.

The $M V$ approach is consistent with the von Neumann-Morgenstern expected utility theory only if (i) the returns of the assets are normally distributed and consequently it is legitimate to ignore higher moments beginning with third order and (ii) the utility function characterizing the investor's attitude towards risk is quadratic. But there is a plethora of empirical studies showing that portfolio returns are generally not normally distributed. Consequently, in some recent studies, the concept of Mean-Variance trade-off has been extended to include the skewness of return in portfolio selection, see Konno and Suzuki [6], Leung et al. [9], Liu et al. [12], Joro and $\mathrm{Na}$ [5], Briec et al. [2], or the kurtosis, see Maringer and Parpas [14], Lai et al. [7], Jondeau and Rockinger [4] among others and also applied in various practical problems, see Rădulescu et al. [17-19]. Many empirical studies show that investors prefer positive skewness, because it implies a low probability of obtaining a large negative return. It was also observed that an increased diversification leads frequently to skewness loss. In order to integrate the skewness, we use the Mean-Variance-Skewness (MVS) utility: $U_{a, b, c}(\boldsymbol{x})=a E(R)-b \sigma^{2}(R)+c S(R), \quad$ where $S(R)$ is the portfolio skewness, $a>0, b>0$ and $c \geq 0$. The optimal portfolio corresponding to the investor's specific degree of risk aversion $\beta=\frac{b}{a}>0$ and degree of absolute prudence $\gamma=\frac{c}{b} \geq 0$, see Eeckhoudt [3], is the solution of the maximization problem, see Briec et al. [2]: $\max _{\boldsymbol{x} \in X} U^{M V S}(\boldsymbol{x})=E(R)-\beta \sigma^{2}(R)+\gamma S(R)$.

We note that this model is not convex also. It is not necessary for our present purposes to pursue the generalization of this result by taking into account the kurtosis or other higher moments, or to discuss further the possibility of nonexistence of some of these moments. But in general, we can construct an utility function defined over the first $m$ moments of the probability distribution of the underlying random variable, provided all of the first $m$ moments exist and are finite. We remark the general lack of convexity of these models (nonconcavity for max problems). Next we propose a convexification technique for a general differentiable nonlinear programming problem with inequality constraints which includes models like (1) and (2).

\section{Equivalent Transformations of the General Nonconvex Model and Local Convexity of the Lagrangian}

In order to formalize models like (1) and (2) having even more complicated constraints like budget constraints, we consider the general model:

$\left\{\begin{array}{c}\min _{x \in X} f(\boldsymbol{x}) \\ g_{j}(\boldsymbol{x}) \leq c_{j}, j=\overline{1, m},\end{array}\right.$

where $f, g_{j}: R^{n} \rightarrow R, j=\overline{1, m}, \quad$ are twice continuously differentiable functions and $X \subset R^{n}$ is a nonempty closed set. It is well known that the Lagrangian function plays an important role in many optimization problems such as the development of duality theory which is the foundation of primal-dual methods. The properties of Lagrangian function are of great importance for the convergence of these methods. Let

$L(\boldsymbol{x}, \boldsymbol{\lambda})=f(\boldsymbol{x})+\boldsymbol{\lambda}^{T}(g(\boldsymbol{x})-\boldsymbol{c})$,

be the Lagrangian function associated to (3) where $\lambda=\left(\lambda_{1}, \ldots, \lambda_{m}\right)^{T} \geq \mathbf{0}, g=\left(g_{1}, \ldots, g_{m}\right)$.

The success of the primal-dual method has been limited to the case when $L(\boldsymbol{x}, \lambda)$ is locally convex at the optimal solution of problem (3). But convexity is not an inherent property in optimization. A set could be nonconvex in one representation space, while the same set could become convex when changing the coordinates 
of the representation space. Let $x^{*}$ satisfy condition (C1): $\boldsymbol{x}^{*}$ is a regular point of the constraints in (3) i.e. $\nabla g_{j}\left(\boldsymbol{x}^{*}\right), j \in J\left(\boldsymbol{x}^{*}\right)$, are linearly independent where $J\left(\boldsymbol{x}^{*}\right)=\left\{j \mid g_{j}(\boldsymbol{x})=c_{j}\right\}$. We also assume condition (C2) to be fulfilled: $x^{*}$ satisfies the second-order sufficiency condition. Then there exists a vector of Lagrange multipliers $\lambda^{*}=\left(\lambda_{1}^{*}, \ldots, \lambda_{m}^{*}\right) \geq \mathbf{0}$ such that

$\left\{\begin{array}{c}\nabla L\left(\boldsymbol{x}^{*}, \lambda^{*}\right)=0, \\ \lambda_{j}^{*}\left(g_{j}\left(\boldsymbol{x}^{*}\right)-c_{j}\right)=0, j=\overline{1, m}\end{array}\right.$ and

$\boldsymbol{y}^{T} \nabla^{2} L\left(\boldsymbol{x}^{*}, \boldsymbol{\lambda}^{*}\right) \boldsymbol{y}>0, \forall \boldsymbol{y} \neq 0$ and

$\boldsymbol{y} \in T\left(\boldsymbol{x}^{*}\right)=\left\{\boldsymbol{y} \in R^{n} \mid \boldsymbol{y}^{T} \nabla g_{j}\left(\boldsymbol{x}^{*}\right)=0, j \in J^{+}\left(\boldsymbol{x}^{*}\right)\right\}$,

where $J^{+}\left(\boldsymbol{x}^{*}\right)=\left\{j \mid \lambda_{j}^{*}>0\right\}$.

The third assumption regarding $x^{*}$ is: (C3) $J^{+}\left(\boldsymbol{x}^{*}\right) \neq \varnothing$. Throughout this paper we consider $h: R \rightarrow R$ a strictly increasing function locally convex in $\boldsymbol{y}^{*}=f\left(\boldsymbol{x}^{*}\right)$. Moreover, without restricting the generality, we suppose that $h$ is a positive function and thus so it is $h^{a}$ and $h^{b}$, where $a, b \geq 1$. We consider the model

$\left\{\begin{array}{c}\min _{\boldsymbol{x} \in X} h^{a}(f(\boldsymbol{x})) \\ h^{b}\left(g_{j}(\boldsymbol{x})\right) \leq d_{j}, j=\overline{1, m},\end{array}\right.$

where $d_{j}=h^{b}\left(c_{j}\right), j=\overline{1, m}$. For $\boldsymbol{x} \in X$ let

$L_{h, a, b}(\boldsymbol{x}, \boldsymbol{\eta})=h^{a}(f(\boldsymbol{x}))+\sum_{j=1}^{m} \eta_{j}\left(h^{b}\left(g_{j}(\boldsymbol{x})\right)-d_{j}\right)$

be the Lagrangian function associated to problem (3) where $\eta=\left(\eta_{1}, \ldots, \eta_{m}\right)^{T} \geq \mathbf{0}$. The optimal Lagrangian multipliers are 0 if $j \notin J^{+}\left(\boldsymbol{x}^{*}\right)$ and

$\eta_{j}^{*}=\frac{\lambda_{j}^{*} a h^{a-1}\left(\boldsymbol{y}^{*}\right) h^{\prime}\left(\boldsymbol{y}^{*}\right)}{b h^{b-1}\left(c_{j}\right) h^{\prime}\left(c_{j}\right)}$, if $j \in J^{+}\left(\boldsymbol{x}^{*}\right)$.

Theorem 1. If $\boldsymbol{x}^{*}$ is a local optimal solution of (3) satisfying (C1)-(C3), then there exist $A>0$ and $B>0$ such that the Hessian matrix $\nabla^{2} L_{h, a, b}\left(\boldsymbol{x}^{*}, \boldsymbol{\eta}^{*}\right)$ is positive definite for $a>A$ and $b>B$.

Proof The Hessian matrix at $\left(x^{*}, \eta^{*}\right)$ is: $\nabla^{2} L_{h, a, b}\left(\boldsymbol{x}^{*}, \boldsymbol{\eta}^{*}\right)=a h^{a-1}\left(\boldsymbol{y}^{*}\right) h^{\prime}\left(\boldsymbol{y}^{*}\right)$.

$\left[\nabla^{2} L\left(\boldsymbol{x}^{*}, \lambda^{*}\right)+(s+(a-1) t) \nabla f\left(\boldsymbol{x}^{*}\right)\left(\nabla f\left(\boldsymbol{x}^{*}\right)\right)^{T}+\right.$

$\left.+\sum_{j=1}^{m}\left(s_{j}+(b-1) t_{j}\right) \nabla f_{j}\left(\boldsymbol{x}^{*}\right)\left(\nabla f_{j}\left(\boldsymbol{x}^{*}\right)\right)^{T}\right]$,

where $s=h^{\prime \prime}\left(\boldsymbol{y}^{*}\right) / h^{\prime}\left(\boldsymbol{y}^{*}\right)>0, t=h^{\prime}\left(\boldsymbol{y}^{*}\right) / h\left(\boldsymbol{y}^{*}\right)>0$, for $j \in J^{+}\left(\boldsymbol{x}^{*}\right): \quad s_{j}=\lambda_{j}^{*} h^{\prime \prime}\left(c_{j}\right) / h^{\prime}\left(c_{j}\right)>0 \quad$ and $t_{j}=\lambda_{j}^{*} h^{\prime}\left(c_{j}\right) / h\left(c_{j}\right)>0$. We note that when the tangent space $T\left(\boldsymbol{x}^{*}\right)$ reduces to the set $\{0\}$, it is straightforward that $\nabla^{2} L_{h, a, b}\left(\boldsymbol{x}^{*}, \eta^{*}\right)$ is positive definite on the entire space $R^{n}$. When $T\left(\boldsymbol{x}^{*}\right) \neq\{\boldsymbol{0}\}$ there is at least one vector $y \neq 0$ such that, for all $j \in J^{+}\left(x^{*}\right)$, the angle between $\boldsymbol{y}$ and $\nabla g_{j}\left(\boldsymbol{x}^{*}\right)$ is $\pi / 2$. If we note $\mathrm{B}=\left\{\boldsymbol{y} \in R^{n} \mid\|\boldsymbol{y}\|=1\right\}, \zeta=\min _{\boldsymbol{y} \in \mathrm{B}} \boldsymbol{y}^{T} \nabla^{2} L\left(\boldsymbol{x}^{*}, \lambda^{*}\right) \boldsymbol{y}$ and $v=\min _{\boldsymbol{y} \in T\left(\boldsymbol{x}^{*}\right) \cap \mathrm{B}} \boldsymbol{y}^{T} \nabla^{2} L\left(\boldsymbol{x}^{*}, \lambda^{*}\right) \boldsymbol{y}>0$, we have $\boldsymbol{y}^{T} \nabla^{2} L_{h, a, b}\left(\boldsymbol{x}^{*}, \eta^{*}\right) \boldsymbol{y} \geq$ $\geq a h^{a-1}\left(\boldsymbol{y}^{*}\right) h^{\prime}\left(\boldsymbol{y}^{*}\right) \cdot\left(v+(s+(a-1) t)\left(\boldsymbol{y}^{T} \nabla f\left(\boldsymbol{x}^{*}\right)\right)^{2}\right)$ $\Rightarrow \boldsymbol{y}^{T} \nabla^{2} L_{h, a, b}\left(\boldsymbol{x}^{*}, \eta^{*}\right) \boldsymbol{y}>0$.

We note that for $\boldsymbol{y} \in \mathbf{B}-T\left(\boldsymbol{x}^{*}\right)$ there exists $k \in J^{+}\left(\boldsymbol{x}^{*}\right)$ such that $\measuredangle\left(\boldsymbol{y}, \nabla g_{k}\left(\boldsymbol{x}^{*}\right)\right) \neq \pi / 2$. But $T\left(\boldsymbol{x}^{*}\right) \cap \mathbf{B}$ is compact and hence there exists $\varepsilon \in(0, \pi / 2)$ such that for any $\boldsymbol{y} \in \mathbf{B}$ for which $\measuredangle\left(y, \nabla g_{j}\left(\boldsymbol{x}^{*}\right)\right) \in[\pi / 2-\varepsilon, \pi / 2+\varepsilon]$ for all $j \in J^{+}\left(\boldsymbol{x}^{*}\right)$, we get $\boldsymbol{y}^{T} \nabla^{2} L\left(\boldsymbol{x}^{*}, \boldsymbol{\lambda}^{*}\right) \boldsymbol{y} \geq v / 2$ and $\boldsymbol{y}^{T} \nabla^{2} L_{h, a, b}\left(\boldsymbol{x}^{*}, \eta^{*}\right) \boldsymbol{y} \geq a h^{a-1}\left(\boldsymbol{y}^{*}\right) h^{\prime}\left(\boldsymbol{y}^{*}\right) v / 2>0$.

For $\boldsymbol{y} \in \mathrm{B}$ for which there exists $k \in J^{+}\left(\boldsymbol{x}^{*}\right)$ such that $\measuredangle\left(y, \nabla g_{k}\left(\boldsymbol{x}^{*}\right)\right) \notin[\pi / 2-\alpha, \pi / 2+\alpha]$ we make the following notations: $\gamma=\min \{|\cos \beta||| \beta-\pi / 2 \mid \geq \alpha, \beta \in[0, \pi]\}$, $\xi=\min _{j \in J^{+}\left(x^{*}\right)}\left\|\nabla f_{j}\left(\boldsymbol{x}^{*}\right)\right\|>0, \quad \underline{s}=\min _{j \in J^{+}\left(\boldsymbol{x}^{*}\right)} s_{j}>0$, $\underline{t}=\min _{j \in J^{+}\left(x^{*}\right)} t_{j}>0$. We find that $\boldsymbol{y}^{T} \nabla^{2} L_{h, a, b}\left(\boldsymbol{x}^{*}, \eta^{*}\right) \boldsymbol{y} \geq a h^{a-1}\left(\boldsymbol{y}^{*}\right) h^{\prime}\left(\boldsymbol{y}^{*}\right)$. $\cdot\left(\zeta+(s+(a-1) t)\left(\boldsymbol{y}^{T} \nabla f\left(\boldsymbol{x}^{*}\right)\right)^{2}+(\underline{s}+(b-1) \underline{t}) \xi^{2} \gamma^{2}\right)$. 
We have $\boldsymbol{y}^{T} \nabla^{2} L_{h, a, b}\left(\boldsymbol{x}^{*}, \eta^{*}\right) \boldsymbol{y}>0$ for all $a \geq A$, $A=A(\boldsymbol{y})=\left\{\begin{array}{c}1, \text { if } \zeta=0 \\ 1-\zeta /\left(t\left(\boldsymbol{y}^{T} \nabla f\left(\boldsymbol{x}^{*}\right)\right)^{2}\right), \text { if } \zeta \neq 0\end{array}\right.$ and $b \geq B=1-\zeta /\left(t \xi^{2} \gamma^{2}\right) . \quad$ Q.E.D.

Let $D_{h, a, b}(\boldsymbol{\eta})=\min _{\boldsymbol{x} \in X} L_{h, a, b}(\boldsymbol{x}, \boldsymbol{\eta}) \quad$ be the dual function of (3) where the minimization is considered locally in a neighborhood of $\boldsymbol{x}^{*}$. Inserting the results from Theorem 1 into the Local Duality Theorem of Xu [21], we can prove:

Theorem 2. If the previous conditions are satisfied and $\boldsymbol{x}^{*}$ is a local optimum of (3), then there exist $A>0$ and $B>0$ such that the dual problem $\max _{\eta \geq 0} D_{h, a, b}(\eta)$ has a local solution $\eta^{*}$ defined by (6), having the optimal value $h^{a}\left(f\left(\boldsymbol{x}^{*}\right)\right)$ and $L_{h, a, b}\left(\boldsymbol{x}^{*}, \boldsymbol{\eta}^{*}\right)=D_{h, a, b}\left(\eta^{*}\right)$ for all $a>A$ and $b>B$.

\section{Illustrative Examples}

\subsection{Example of real data failing to fulfill} the requirement on the estimated covariance matrix of being positive semi-definite

We consider the practical case of missing data and the construction of the NAN - covariance matrix that may be not positive semi-definite. We exemplify here by considering the following data set given in Table 1 consisting in the returns $r_{1}, r_{2}, r_{3}$ of three assets spanning two weeks (10 working days). Missing data are labeled $\mathrm{NaN}$ (Matlab convention for Not a Number).

Table 1. Historical data set of daily rate of returns $r_{1}, r_{2}, r_{3}$ of three assets.

\begin{tabular}{|c|c|c|c|}
\hline Day $k$ & $r_{k 1}$ & $r_{k 2}$ & $r_{k 3}$ \\
\hline 1 & $-0,5$ & $-0,38$ & -1 \\
\hline 2 & 0 & 0,21 & $-0,52$ \\
\hline 3 & 0,1 & 0,21 & 0 \\
\hline 4 & $-0,261$ & $\mathrm{NaN}$ & $-0,43$ \\
\hline 5 & 0 & $-0,21$ & 0,198 \\
\hline 6 & 0,18 & $\mathrm{NaN}$ & 0,31 \\
\hline 7 & 0,02 & 0,215 & 0,24 \\
\hline 8 & $\mathrm{NaN}$ & 0,048 & 0,09 \\
\hline 9 & $\mathrm{NaN}$ & 0,02 & 0,13 \\
\hline 10 & 0,089 & $-0,23$ & 0,068 \\
\hline
\end{tabular}

Starting from $\boldsymbol{r}_{i}^{T}=\left(r_{1 i}, \ldots, r_{10, i}\right)$ we calculate the NAN-covariance matrix denoted by $\widetilde{\Sigma}=\left(\widetilde{\Sigma}_{i j}\right)_{1 \leq i, j \leq 3}$, by ignoring the missing data. We suppose that for every $i, j \in\{1,2,3\}$ there is at least $k \in\{1, \ldots, 10\}$ such that both $r_{k i}$ and $r_{k j}$ are known.

$\tilde{\Sigma}_{i j}=\frac{\sum_{k=1}^{10} \varepsilon_{k i} \varepsilon_{k j}\left(\tilde{r}_{k i}-\mu_{i}\right)\left(\tilde{r}_{k j}-\mu_{j}\right)}{\sum_{k=1}^{10} \varepsilon_{k i} \varepsilon_{k j}}$,

$\tilde{r}_{k i}=\left\{\begin{array}{l}0, \text { if } r_{k i} \text { is not known }(\mathrm{NaN}) \\ r_{k i}, \text { otherwise }\end{array}\right.$,

$\varepsilon_{k i}=\left\{\begin{array}{l}0, \text { if } r_{k i} \text { is not known }(\mathrm{NaN}) \\ 1, \text { otherwise, }\end{array}\right.$,

$\mu_{i}=\frac{\sum_{k=1}^{10} \tilde{r}_{k i} \varepsilon_{k i}}{\sum_{k=1}^{10} \varepsilon_{k i}}$,

for all $1 \leq i, j \leq 3$ and $1 \leq k \leq 10$.

The resulting NAN-covariance matrix is:

$$
\widetilde{\Sigma}=\left(\begin{array}{lll}
0.050221 & 0.037172 & 0.089450 \\
0.037172 & 0.053831 & 0.037213 \\
0.089450 & 0.037213 & 0.177143
\end{array}\right) \text {. }
$$

The corresponding eigenvalues are:

$$
\Lambda=\left(\begin{array}{l}
-0.00172 \\
0.045771 \\
0.237144
\end{array}\right)
$$

and thus, $\tilde{\Sigma}$ is not positive semi-definite.

There are methods for estimating covariance or correlation matrices which ensure that the matrix is positive definite or at least positive semi-definite, see Schottle and Werner [20], Jaeckle and Rebonato [22] for a thoroughly discussion on the methods for creating a valid covariation/correlation matrix.

But the matrices obtained with these methods must be verified because they may distort the initial information even if they become symmetric and positive semi-definite.

The advantage of the convexification method proposed is that the minimum variance model with the NAN-covariance matrix calculated by ignoring the missing data can be used.

\subsection{Example of local convexification of the Lagrangian function}

We present an example which illustrates the local convexification of the Lagrangian function. This example gives a geometrical 
illustration of Theorem 1 and 2. We consider the following model

$$
\left\{\begin{array}{c}
\min f(\boldsymbol{x})=1-2 x_{1} x_{2} \\
2 x_{1}+4 x_{2} \leq 1 \\
0 \leq x_{1}, x_{2} \leq 1
\end{array}\right.
$$

Note that the objective function is not convex.

The optimal solution of this problem that is $\boldsymbol{x}^{*}=\left(\frac{1}{4}, \frac{1}{8}\right)$ is located on the boundary of the feasible region, $y^{*}=f\left(x^{*}\right)=\frac{15}{16}$.

The Lagrangian function of model (7) is

$$
L(\boldsymbol{x}, \lambda)=1-2 x_{1} x_{2}+\lambda\left(2 x_{1}+4 x_{2}-1\right) .
$$

The optimal multiplier is $\lambda^{*}=\frac{1}{8}>0$ and the Hessian of the Lagrangian function is

$$
\nabla^{2} L\left(x^{*}, \lambda^{*}\right)=\nabla^{2} L\left(\left(\frac{1}{4}, \frac{1}{8}\right), \frac{1}{8}\right)=\left(\begin{array}{cc}
0 & -2 \\
-2 & 0
\end{array}\right)
$$

which is an indefinite matrix. Now we consider the transformed model (4) with

$$
\left\{\begin{array}{l}
h(x)=e^{x} \\
a=1 \\
b=4
\end{array}\right.
$$

and the Lagrangian defined in (5) is

$$
L_{h, 1,4}(\boldsymbol{x}, \eta)=e^{1-2 x_{1} x_{2}}+\eta\left(e^{4\left(2 x_{1}+4 x_{2}\right)}-e^{4}\right) .
$$

Using (6), we find $\eta^{*}=\frac{1}{32} e^{-\frac{49}{16}}$. The new Hessian matrix is

$$
\nabla^{2} L_{h, 1,4}\left(x^{*}, \eta^{*}\right)=\left(\begin{array}{cc}
\frac{33}{16} e^{\frac{15}{16}} & \frac{17}{8} e^{\frac{15}{16}} \\
\frac{17}{8} e^{\frac{15}{16}} & \frac{33}{4} e^{\frac{15}{16}}
\end{array}\right)
$$

which is a positive definite matrix. The level curves of $L\left(\boldsymbol{x}, \lambda^{*}\right)$ and $L_{h, 1,4}\left(\boldsymbol{x}, \eta^{*}\right)$ are given in Figures 1 respectively 2 . As we can see, we obtain the local convexity in a neighborhood of the optimal point of contours of the Lagrangian function of model (4).

\section{Conclusions}

In this paper we have shown how to locally convexify the Lagrangian function of a nonconvex optimization problem. Thus, we have extended the class of optimization problems to which dual methods can be applied. Specifically, we have proved that, under mild assumptions, the Hessian of the

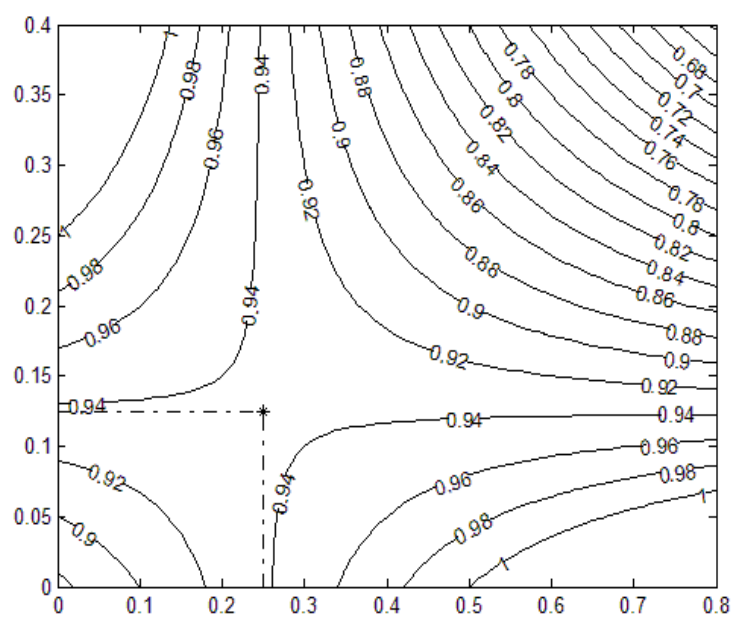

Figure 1. Contour levels of the Lagrangian function $L\left(\boldsymbol{x}, \lambda^{*}\right)$.

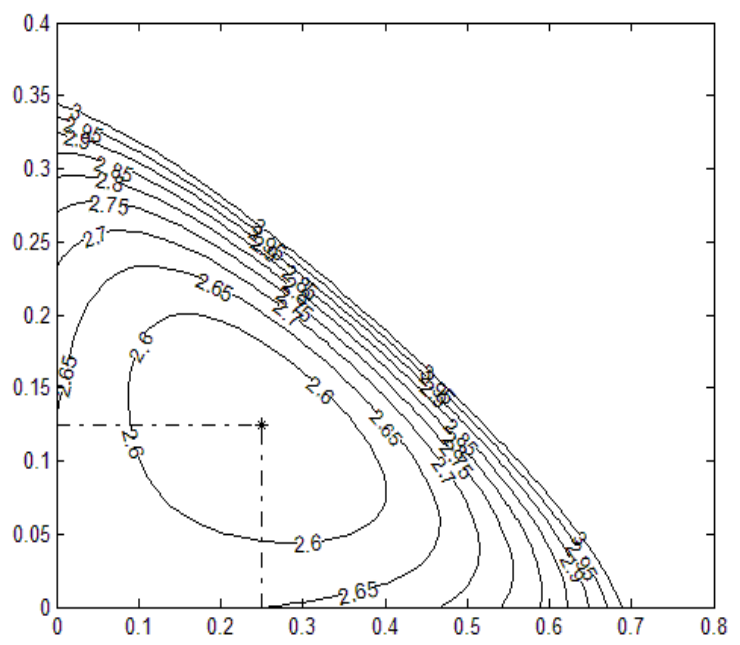

Figure 2. Contour levels of the Lagrangian function $L_{h, 1,4}\left(\boldsymbol{x}, \eta^{*}\right)$ of the equivalent model.

Lagrangian function in some transformed equivalent problem formulations becomes positive definite in a neighborhood of a local optimal point of the original problem. From local duality theory, convexity in the Lagrangian guarantees the success of the dual search and zero duality gap is thus guaranteed when the primal-dual method is applied to the constructed equivalent form. Practical classes of problems where the proposed method can be applied include portfolio selection models. A numerical example which illustrates the main results is given.

\section{Acknowledgements}

This work was supported by a grant of the Romanian National Authority for Scientific Research, CNCS -- UEFISCDI, project number PN-II-ID-PCE-2011-3-0908. 


\section{REFERENCES}

1. BERTSEKAS, D., Convexification Procedures and Decomposition Methods for Nonconvex Optimization Problems, J. of Opt. Theory and App., vol. 29, 1979, pp. 169-197.

2. BRIEC W., K. KERSTENS, J. LESOURD, Single-Period Markowitz Portfolio Selection, Performance Gauging, and Duality: A Variation on the Luenberger Shortage Function. J. of Opt. Theory and App., vol. 120, No. 1, 2004, pp. 1-27.

3. EECKHOUDT, L., C. GOLLIER, H. SCHLESINGER, Economic and Financial Decisions under Risk, Princeton University Press, Princeton and Oxford, 2005.

4. JONDEAU, E., M. ROCKINGER Optimal Portfolio Allocation under Higher Moments, European Fin. Man., vol. 12(1), 2006, pp. 29-55.

5. JORO, T., P. NA, Portfolio Performance Evaluation in a Mean--Variance-Skewness Framework. European J. of Op. Res., vol. 175(1), 2006, pp. 446-461.

6. KONNO, H., K. SUZUKI, A MeanVariance-Skewness Portfolio Optimization Model. J. of the Op. Res. Society of Japan vol. 38(2), 1995, pp. 173-187.

7. LAI, K. K., Y. LEAN, W. SHOUYANG, Mean-Variance-Skewness-Kurtosis-based Portfolio Optimization, Proc. of $1^{\text {st }}$ Intl. Multi-Symposiums on Computer and Computational Sciences, 2006, pp. 1-6.

8. LASDON, L. S., Optimization Theory for Large Systems. Macmillan Company, London, England, 1970.

9. LEUNG, M. T., H. DAOUK, A. S. CHEN, Using Investment Portfolio Return to Combine Forecasts: a Multiobjective Approach. European J. of Op. Res., vol. 134, 2001, pp. 84-102.

10. LI, D., Zero Duality Gap for a Class of Nonconvex Optimization Problems. Journal of Optimization Theory and Applications, vol. 85, 1995, pp. 309-324.

11. LI, D., Saddle-Point Generation in Nonlinear Nonconvex Optimization. Nonlinear Analysis, Theory, Methods and Applications, vol. 30, 1997, pp. 4339-4344.
12. LIU, S. C., S. Y. WANG, W. H. QIU, A Mean-Variance-Skewness Model for Portfolio Selection with Transaction Costs. International Journal of Systems Sciences, vol. 34(4), 2003, pp. 255-262.

13. LUENBERGER, D. G., Linear and Nonlinear Programming, $2^{\text {nd }}$ ed., AddisonWesley, Reading, Massachusetts, 1984.

14. MARINGER, D., P. PARPAS, Global Optimization of Higher Order Moments in Portfolio Selection. J. of Global Optimization., vol. 43, 2009, pp. 219-230.

15. MARKOWITZ, H. M. Portfolio Selection, Journal of Finance, vol. 7, 1952, pp. 77-91.

16. MARKOWITZ, H. M. Portfolio Selection. Efficient Diversification of Investments. John Wiley \& Sons, Inc., New York, 1959.

17. RĂDULESCU, M., C. Z. RĂDULESCU, M. TUREK RAHOVEANU, G. ZBĂGANU, A Portfolio Theory Approach to Fishery Management, Studies in Informatics and Control, vol. 19(3), 2010, pp. 285-294.

18. RĂDULESCU, C. Z., M. TUREK RAHOVEANU, A Multi-Criteria Evaluation Framework for Fish Farms, Studies in Informatics and Control, vol. 20(2), 2011, pp. 181-186.

19. RĂDULESCU, C. Z., M. RĂDULESCU, A Decision Support Tool Based on a Portfolio Selection Model for Crop Planning under Risk, Studies in Informatics and Control, ISSN 1220-1766, vol. 21(4), 2012, pp. 377-382.

20. SCHOTTLE, K., R. WERNER, Improving the Most General Methodology to Create a Valid Correlation Matrix, Transactions of the Wessex Institute, Risk Analysis IV, Eds: C. A. Brebbia, 2004, pp. 701-710, DOI: 10.2495/RISK040641.

21. XU, Z. K., Local Saddle Points and Convexification for Nonconvex Optimization Problems. Journal of Optimization Theory and Applications, vol. 94, 1997, pp. 736-746.

22. JAECKEL, P., R. REBONATO, The Most General Methodology for Creating a Valid Correlation Matrix for Risk Management and Option Pricing Purposes. Journal of Risk, vol. 2(2), 2000. 